\title{
Surgical strategy for intraductal papillary mucinous neoplasms of the pancreas
}

\author{
Seiko Hirono ${ }^{1} \cdot$ Hiroki Yamaue $^{1}$
}

Received: 2 October 2019 / Accepted: 8 October 2019 / Published online: 5 December 2019

(c) The Author(s) 2019

\begin{abstract}
The current treatment strategy for intraductal papillary mucinous neoplasms (IPMNs), based on the international consensus guideline, has been accepted widely. However, reported outcomes after surgical resection for IPMN show that once the tumor progresses to invasive intraductal papillary mucinous carcinoma (IPMC), recurrence is not uncommon. The surgical treatment for IPMN is invasive and sometimes followed by complications. Therefore, the best timing for resection might be at the point when high-grade dysplasia (HGD) is evident. According to previous reports, main duct type IPMN has a high malignant potential and its surgical resection is universally accepted, whereas, the incidence of HGD/invasive IPMC in branch duct and mixed type IPMNs is thought to be lower. In addition to mural nodules and a dilated main pancreatic duct, cytology and measurement of the carcinoembryonic antigen level in the pancreatic juice might be useful to differentiate HGD/ invasive IPMC from low-grade dysplasia. The nomogram proposed recently to predict the risk of HGD/invasive IPMC in IPMN patients might help surgeons decide on the best treatment strategy, depending on the patient's age and general condition. Second resection for high-risk lesions in the remnant pancreas might improve the survival of IPMN patients.
\end{abstract}

Keywords IPMN $\cdot$ CEA in the pancreatic juice $\cdot$ Mural nodule $\cdot$ High-grade dysplasia $\cdot$ Invasive IPMC

\section{Introduction}

The incidence of intraductal papillary mucinous neoplasm (IPMN) of the pancreas has increased recently in line with awareness and advances in radiographic and endoscopic imaging. IPMNs exhibit a spectrum of neoplastic transformation, classified as low-grade dysplasia (LGD), highgrade dysplasia (HGD), and invasive intraductal papillary carcinoma (IPMC) [1,2]. The following three guidelines for the diagnosis and management of IPMNs have been used widely: The international consensus guideline (ICG) $[1,3,4]$, the European evidence-based guideline [5], and the American Gastroenterological Association (AGA) Institute guideline [6]. There appears to be a recent trend toward an increase in the number of IPMN patients being followed up but not undergoing surgical resection for three reasons: First, the number of patients with IPMN diagnosed early is

Seiko Hirono

seiko-h@wakayama-med.ac.jp

1 Second Department of Surgery, School of Medicine, Wakayama Medical University, 811-1 Kimiidera, Wakayama 641-8510, Japan increasing with advances in radiographic and endoscopic imaging [7-10]; second, the operative treatment is invasive; and third, the frequency of postoperative complications after pancreatectomy is higher than that after most other gastrointestinal operations. However, in some patients, IPMN may become invasive during follow-up, and recurrence can develop even after surgery, with progression to unresectable invasive IPMC from metastases or local spread. To avoid these miserable outcomes, it would be advantageous to identify when IPMN should be resected and which factors are significantly associated with the appropriate surgical indications for IPMN.

Recent studies have demonstrated the malignant progression of IPMN and that pancreatic ductal adenocarcinoma (PDAC), being a high-risk lesion, may develop in the remnant pancreas after surgical resection for IPMN [11-15]. It is important that we evaluate what treatment could improve the survival of these patients with high-risk lesions in the remnant pancreas after surgery for IPMN. In this article, we review which patients would gain oncological benefits from surgical resection for IPMN and what operative procedures are appropriate. 


\section{Surgical indication of IPMN based on outcomes after resection}

According to previous reports, the frequency of overall recurrence after surgical resection for noninvasive IPMN (LGD/HGD) ranges from 0 to $17 \%$ [11, 12, 15-17]. In a Japanese multicenter study of 1074 IPMN patients who underwent surgery, recurrence developed after surgery in $0.7 \%$ of 827 patients with LGD/HGD, but in $43.3 \%$ of 247 patients with invasive IPMC [11]. Thus, once the tumor progresses to having an invasive component, recurrence is likely, even after surgery $[11,12,18]$. However, pancreatectomy for IPMN is invasive, despite advanced and optimally safe operative techniques. Therefore, if a tumor is diagnosed as LGD IPMN by radiographic and endoscopic findings, it could be monitored without surgery. In light of these results, HGD might be the best timing for surgical resection for IPMN.

The revised ICGs in 2012 and 2017 defined that malignant IPMN includes only invasive IPMC, but not HGD $[1,3]$, and this definition could confuse clinicians about the surgical indication, HGD and invasive IPMC (HGD/ invasive IPMC), or only invasive IPMC. In consideration of the poor prognosis after surgery for invasive IPMC, we believe that HGD/invasive IPMC is the surgical indication, and it is important to identify the predictive factors associated with HGD/invasive IPMC.

\section{Predictive factors associated with HGD/invasive IPMC}

Depending on the morphology of the ductal system, IPMNs are classified into three types: main duct (MD) type, branch duct (BD) type, and mixed type [1, 3-5]. In MD type IPMNs, the frequency of HGD/invasive IPMC ranges from 36 to $100 \%$ and the frequency of invasive IPMC ranges from 11 to $81 \%$ [16, 19-28]. Considering these high incidences of HGD/invasive IPMC, surgical resection for MD type IPMN is universally accepted. Conversely, in $\mathrm{BD} /$ mixed-type IPMNs, the incidence of HGD/invasive IPMC ranges from 14.4 to $47.9 \%$, and the incidence of invasive IPMC ranges from 6.1 to $37.7 \%$ [29-35]. Thus, the frequency of HGD/invasive IPMC in $\mathrm{BD} /$ mixed-type IPMNs is significantly lower than those in MD type IPMN, and we should consider the predictive factors associated with this.

The ICG revised in 2017 described three factors as high-risk stigmata; namely, obstructive jaundice, enhancing of a mural nodule $\geq 5 \mathrm{~mm}$, and a main pancreatic duct (MPD) $\geq 10 \mathrm{~mm}$; and that any of these high-risk factors should be an absolute surgical indication [1]. Furthermore, endoscopic ultrasonography (EUS) is recommended for patients with any of the following nine features of concern: pancreatitis, cyst $\geq 3 \mathrm{~cm}$, enhancing mural nodule $<5 \mathrm{~mm}$, thickened/enhancing cyst wall, MPD 5-9 mm, abrupt change in caliber or pancreatic duct with distal pancreatic atrophy, lymphadenopathy, increased serum carbohydrate antigen 19-9 (CA19-9) level, and a cyst growth rate $\geq 5 \mathrm{~mm} / 2$ years. Subsequently, any of the three following findings by EUS would define surgical indication: mural nodule $\geq 5 \mathrm{~mm}$, MPD features suspicious for involvement, and positive cytology [1].

The European guideline suggested that the absolute indications for surgery are positive cytology, a solid mass, tumor-related jaundice, enhancing mural nodules $\geq 5 \mathrm{~mm}$, and MPD $\geq 10 \mathrm{~mm}$. These factors are similar to the highrisk stigmata in ICG. This guideline also suggests the following relative indications: a growth rate $\geq 5 \mathrm{~mm} / \mathrm{year}$, an increased serum CA19-9 level, MPD 5-9.9 mm, cyst diameter $\geq 40 \mathrm{~mm}$, onset of diabetes mellitus, acute pancreatitis caused by IPMN, and enhancing mural nodules $<5 \mathrm{~mm}$ [5]. It recommends surgery for patients without significant comorbidities and one or more relative indication for surgery and for patients with significant co-morbidities and two or more relative indications for surgery [5].

The AGA guideline suggests that both a solid component and a dilated MPD and/or concerning features on EUS and FNA are indications for surgery [6]. According to these guidelines, which are based on evidence from many reports, mural nodule and dilated MPD on radiographic image, and obstructive jaundice or pancreatitis caused by IPMN are important indications for surgery.

In addition to a mural nodule and dilated MPD, several studies have found that cytology and/or tumor markers, including carcinoembryonic antigen (CEA) in the cystic fluid obtained by EUS-guided fine needle aspiration (EUSFNA), or in the pancreatic juice obtained from MPD by endoscopic retrograde pancreatography (ERP), were useful to establish surgical indication for IPMN [36-41]. The diagnostic accuracy of cystic fluid or pancreatic juice cytology for HGD/invasive IPMC is poor because the sensitivity rate is low [36-38]. However, the positive predictive value (PPV) of cytology for HGD/invasive IPMC is excellent and might be a useful method to determine surgical indication [36, 37]. Several studies have reported the usefulness of measuring the CEA level in pancreatic juice obtained from MPD by ERP to differentiate LGD from HGD/invasive IPMC [18, 38-40]. However, there are few reports documenting the usefulness of measuring the CEA level in cystic fluid obtained by EUS-FNA [41]. We consider that BD-type IPMNs often consist of multilocular cysts, but it is unknown which cyst has the most severe atypia, whereas the pancreatic juice in the MPD obtained by ERP included secreted CEA from all of the pancreatic ducts. That is why 
measurement of the CEA level in pancreatic juice might be more useful than measuring that in the cystic fluid. Furthermore, EUS-FNA for IPMN carries a theoretical risk of peritoneal seeding, and there have been several reports of this [42, 43], whereas obtaining pancreatic juice by ERP is not associated with a risk of peritoneal seeding. Nevertheless, the ICG do not recommend routine ERP for collecting pancreatic juice during evaluation of the malignant potential of an IPMN because the procedure is invasive and could cause post-ERP pancreatitis [1,3]. Based on these findings, evaluation of the cytology or measurement of the CEA level in pancreatic juice obtained by ERP is helpful for determining the surgical indication for IPMN, whereas because ERP is an invasive procedure, it should be performed only as a diagnostic adjunct, not as a routine examination.

Recent large-scale studies have proposed using a nomogram to predict the individual risk of HGD/invasive IPMC in patients with IPMN [44-47]. These models show the diagnostic ability of HGD/invasive IPMC, and are contributory to determining the best treatment strategy, depending on the patient's age and general condition.

\section{Treatment strategy for invasive IPMC}

According to a Japanese multicenter study of 1074 IPMN patients, $30.8 \%$ of 247 patients with invasive IPMC treated by surgery had lymph node metastases, including $2.7 \%$ of 74 with T1a invasive IPMC with a depth of invasive component of $\leq 5 \mathrm{~mm}$ [11]. This is consistent with other reports [12, 15, 16]. Based on these findings, lymph node dissection same as that done for PDAC might be necessary for pancreatectomy for invasive IPMC to improve survival. Therefore, it is important to identify the predictive factors associated with invasive IPMC to decide on the most appropriate operative procedure. We reported previously that a large mural nodule was an independent predictor of invasive IPMC in all types of IPMNs (MD, BD, and mixed types), and a high CEA level in the pancreatic juice was an independent factor of invasive IPMC in MD and mixed-type IPMNs [48]. The diagnostic accuracy using our predictors was significantly higher than that using high-risk stigmata or features of concern in the ICG $[3,48]$. Evaluation of mural nodule size and CEA level in the pancreatic juice might be useful to predict the appropriate degree of lymph node dissection during pancreatectomy, preoperatively.

The oncological benefits of postoperative adjuvant therapy for invasive IPMC remain controversial. Some studies have found that postoperative adjuvant therapy does not impact on the survival of patients with invasive IPMC [11, 49]. Other studies have found that postoperative adjuvant therapy could improve the survival of patients with invasive IPMC patients with advanced stage or lymph node metastases [50, 51]. To confirm the oncological benefits of postoperative adjuvant therapy for invasive IPMC patients, a large-scale prospective study is necessary.

\section{Optimal operative procedures for noninvasive IPMN}

Patients with noninvasive IPMN are expected to survive long-term; therefore, quality of life, including endocrine and exocrine function of the remnant pancreas after surgery, must be considered. Several studies have reported the feasibility of IPMN enucleation, but this is controversial [52-54]. While tumor enucleation can preserve most pancreatic parenchyma, resulting in long-term good pancreatic function, performing this procedure for IPMN carries a risk of rupturing the cysts during the operation, causing peritoneal seeding. Moreover, it is frequently associated with postoperative complications including pancreatic fistula.

For noninvasive IPMN located in the pancreatic body, several investigators have reported better long-term outcomes after central pancreatectomy than after distal pancreatectomy [55-57]. Furthermore, recent reports have shown that laparoscopic or robotic pancreatectomy for IPMN is feasible and minimally invasive [58-60]. Establishing an appropriate operative procedure for noninvasive IPMN from the viewpoint not only of short-term outcomes, including postoperative complications, but also of long-term outcomes, including preserving function of the remnant pancreas, is critical.

\section{Treatment strategy for high-risk lesions in the remnant pancreas after surgical resection for IPMN}

According to the Japanese multicenter study of 1074 IPMN patients, the 5-year and 10-year cumulative incidences of high-risk lesions; namely, malignant progression of IPMN and new development of PDAC, in the remnant pancreas after surgical resection for IPMN, were $6.2 \%$ and $12.6 \%$, respectively [11]. Furthermore, second surgery for metachronous high-risk lesions in the remnant pancreas seemed to improve survival [11]. Based on these results, lifelong surveillance of the remnant pancreas following surgery might be necessary for IPMN patients, to enable us to diagnose and treat high-risk lesions in the remnant pancreas at an early stage.

\section{Conclusions}

To improve the survival of IPMN patients, resection might be necessary before the tumor progresses to having invasive components. Evaluation of the mural nodule and MPD size, in addition to symptoms including obstructive jaundice and pancreatitis, will help to determine the surgical indication 
for IPMN. Furthermore, measurement of the CEA level in the pancreatic juice might be useful to determine the surgical indication and the most appropriate operative procedure. High-risk lesions in the remnant pancreas, such as malignant progression of IPMN or new development of PDAC, could develop over 5 years, and then lifelong surveillance after surgery is necessary. Second surgery for high-risk lesions in the remnant pancreas might also improve survival.

\section{Compliance with ethical standards}

Conflict of interest We have no financial interests or potential conflicts of interest to declare.

Open Access This article is licensed under a Creative Commons Attribution 4.0 International License, which permits use, sharing, adaptation, distribution and reproduction in any medium or format, as long as you give appropriate credit to the original author(s) and the source, provide a link to the Creative Commons licence, and indicate if changes were made. The images or other third party material in this article are included in the article's Creative Commons licence, unless indicated otherwise in a credit line to the material. If material is not included in the article's Creative Commons licence and your intended use is not permitted by statutory regulation or exceeds the permitted use, you will need to obtain permission directly from the copyright holder. To view a copy of this licence, visit http://creativecommons.org/licenses/by/4.0/.

\section{References}

1. Tanaka M, Fernández-del Castillo C, Kamisawa T, Jang JY, Levy $\mathrm{P}$, Ohtsuka T, et al. Revisions of international consensus Fukuoka guidelines for the management of IPMN of the pancreas. Pancreatology. 2017;17:738-53.

2. Basturk O, Hong SM, Wood LD, Adsay NV, Albores-Saavedra $\mathrm{J}$, Biankin AV, et al. A revised classification system and recommendations from the Baltimore Consensus Meeting for neoplastic precursor lesions in the pancreas. Am J Surg Pathol. 2015;39:1730-41.

3. Tanaka M, Fernández-del Castillo C, Adsay V, Chari S, Falconi M, Jang JY, et al. International consensus guidelines 2012 for the management of IPMN and MCN of the pancreas. Pancreatology. 2012;12:183-97.

4. Tanaka M, Chari S, Adsay V, Fernandez-del Castillo C, Falconi M, Shimizu M, et al. International consensus guidelines for management of intraductal papillary mucinous neoplasms and mucinous cystic neoplasms of the pancreas. Pancreatology. 2006;6:17-32.

5. The European Study Group on Cystic Tumours of the Pancreas. European evidence-based guidelines on pancreatic cystic neoplasms. Gut. 2018;67:789-804.

6. Vege SS, Ziring B, Jain R, Moayyedi P, American Gastroenterology Association. American Gastroenterological Association institute guideline on the diagnosis and management of asymptomatic neoplastic pancreatic cysts. Gastroenterology. 2015;148:819-22.

7. Oyama H, Tada M, Takagi K, Tateishi K, Hamada T, Nakai Y, et al. Long-term risk of malignancy in branch duct intraductal papillary mucinous neoplasms. Gastroenterology. 2019. https:// doi.org/10.1053/j.gastro.2019.08.032.
8. Marchegiani G, Andrianello S, Pollini T, Caravati A, Biancotto $\mathrm{M}$, Secchettin E, et al. "Trivial" cysts redefine the risk of cancer in presumed branch-duct intraductal papillary mucinous neoplasms of the pancreas: a potential target for follow-up discontinuation? Am J Gastroenterol. 2019. https://doi.org/10.14309/ajg.00000 00000000378.

9. Weaver DT, Berland LL, Knudsen AB, Peters MLB, Hur C, Kong $\mathrm{CY}$, et al. Testing for verification bias in reported malignancy risks for side branch intraductal papillary mucinous neoplasms: a simulation modeling approach. Am J Roentgenol. 2019;212:596-601.

10. Canto MI, Almario JA, Schulick RD, Yeo CJ, Klein A, Blackford $\mathrm{A}$, et al. Rsik of neoplastic progression in individual at high risk for pancreatic cancer undergoing long-term surveillance. Gastroenterology. 2018;155:740-51.

11. Hirono S, Shimizu Y, Ohtsuka T, Kin T, Hara K, Kanno A, et al. Recurrence patterns after surgical resection of intraductal papillary mucinous neoplasm (IPMN) of the pancreas; a multicenter, retrospective study of 1074 IPMN patients by the Japan Pancreas Society. J Gastroentrol. 2019. https://doi.org/10.1007/ s00535-019-01617-2.

12. Hirono S, Kawai M, Okada KI, Miyazawa M, Shimizu A, Kitahata $\mathrm{Y}$, et al. Long-term surveillance is necessary after operative resection for intraductal papillary mucinous neoplasm of the pancreas. Surgery. 2016;160:306-17.

13. Miyasaka Y, Ohtsuka T, Tamura K, Mori Y, Shindo K, Yamada $\mathrm{D}$, et al. Predictive factors for the metachronous development of high-risk lesions in the remnant pancreas after partial pancreatectomy for intraductal papillary mucinous neoplasm. Ann Surg. 2016;263:1180-7.

14. Date K, Ohtsuka T, Fujimoto T, Tamura K, Kimura H, Matsunaga $\mathrm{T}$, et al. Molecular evidence for monoclonal skip progression in main duct intraductal papillary mucinous neoplasms of the pancreas. Ann Surg. 2017;265:969-77.

15. Kang MJ, Jang JY, Lee KB, Chang YR, Kwon W, Kim SW. Long-term prospective cohort study of patients undergoing pancreatectomy for intraductal papillary mucinous neoplasm of the pancreas. Implications for postoperative surveillance. Ann Surg. 2014;260:356-63.

16. Marchegiani G, Mino-Kenudson M, Ferrone CR, Morales-Oyarvide V, Warshaw AL, Lillemoe KD, et al. Patterns of recurrence after resection of IPMN. Who, When, and How? Ann Surg. 2015;262:1108-14.

17. Dhar VK, Merchant NB, Patel SH, Bentrem DJ, LeCompte MT, Idrees K, et al. Does surgical margin impact recurrence in noninvasive intraductal papillary mucinous neoplasms? A multi-institutional study. Ann Surg. 2018;268:469-78.

18. Hirono S, Tani M, Kawai M, Ina S, Nishioka R, Miyazawa M, et al. Treatment strategy for intraductal papillary mucinous neoplasm of the pancreas based on malignant predictive factors. Arch Surg. 2009; 144:345-9 (discussion 349-350).

19. Schmidt CM, White PB, Waters JA, Yiannoutsos CT, Cummings OW, Baker M, et al. Intraductal papillary mucinous neoplasms: predictors of malignant and invasive pathology. Ann Surg. 2007;246:644-51.

20. Nagai K, Doi R, Kida A, Kami K, Kawaguchi Y, Ito T, et al. Intraductal papillary mucinous neoplasms of the pancreas: clinicopathologic characteristics and long-term follow-up after resection. World J Surg. 2008;32:271-8.

21. Hwang DW, Jang JY, Lee SE, Lim CS, Lee KU, Kim SW. Clinicopathologic analysis of surgically proven intraductal papillary mucinous neoplasms of the pancreas in SNUH: a 15-year experience at a single academic institution. Langenbecks Arch Surg. 2012;397:93-102.

22. Waters JA, Schmidt CM, Pinchot JW, White PB, Cummings OW, Pitt HA, et al. CT vs. MRCP: optimal classification of IPMN type and extent. J Gastrointest Surg. 2008;12:101-9. 
23. Crippa S, Fernandez-del Castillo C, Salvia R, Finkelstein D, Bassi C, Domínguez I, et al. Mucin-producing neoplasms of the pancreas: an analysis of distinguishing clinical and epidemiologic characteristics. Clin Gastroenterol Hepatol. 2010;8:213-9.

24. Salvia R, Fernandez-del Castillo C, Bassi C, Thayer SP, Falconi M, Mantovani W, et al. Main-duct intraductal papillary mucinous neoplasms of the pancreas. Ann Surg. 2004;239:678-87.

25. Suzuki Y, Atomi Y, Sugiyama M, Isaji S, Inui K, Kimura W, et al. Cystic neoplasm of the pancreas: a Japanese multiinstitutional study of intraductal papillary mucinous tumor and mucinous cystic tumor. Pancreas. 2004;28:241-6.

26. Schnelldorfer T, Sarr MG, Nagorney DM, Zhang L, Smyrk TC, Qin R, et al. Experience with 208 resections for intraductal papillary mucinous neoplasm of the pancreas. Arch Surg. 2008;143:639-46.

27. Ohno E, Hirooka Y, Itoh A, Ishigami M, Katano Y, Ohmiya N, et al. Intraductal and benign tumors by endoscopic ultrasonography findings of mural nodules. Ann Surg. 2009;249:628-34.

28. Hackert T, Fritz S, Klauss M, Bergmann F, Hinz U, Strobel O, et al. Main-duct intraductal papillary mucinous neoplasm: parameters predictive of progression. Ann Surg. 2014;260:680-8.

29. Sahora K, Mino-Kenudson M, Brugge W, Thayer SP, Ferrone $\mathrm{CR}$, Sahani D, et al. Branch duct intraductal papillary mucinous neoplasms: does cyst size change the tip of the scale? A critical analysis of the revised international consensus guidelines in a large single-institutional series. Ann Surg. 2013;258:466-75.

30. Goh BKP, Thng CH, Tan DM, Low AS, Wong JS, Cheow PC, et al. Evaluation of the Sendai and 2012 international consensus guidelines based on cross-sectional imaging findings performed for the initial triage of mucinous cystic lesions of the pancreas: a single institution experience with 114 surgically treated patients. Am J Surg. 2014;208:202-9.

31. Aso T, Ohtsuka T, Matsunaga T, Kimura H, Watanabe Y, Tamura $\mathrm{K}$, et al. High-risk stigmata of the 2012 international consensus guidelines correlate with the malignant grade of branch duct intraductal papillary mucinous neoplasms of the pancreas. Pancreas. 2014;43:1239-43.

32. Roch AM, Ceppa EP, DeWitt JM, Al-Haddad MA, House MG, Nakeeb A, et al. International consensus guidelines parameters for the prediction of malignancy in intraductal papillary mucinous neoplasm are not properly weighted and are not cumulative. HPB Oxf. 2014;16:929-35.

33. Jang JY, Park T, Lee S, Kang MJ, Lee SY, Lee KB, et al. Validation of international consensus guidelines for the resection of branch duct type intraductal papillary mucinous neoplasms. Br J Surg. 2014;101:686-92.

34. Fritz S, Klauss M, Bergmann F, Strobel O, Schneider L, Werner J, et al. Pancreatic main-duct involvement in branch-duct IPMNs. Ann Surg. 2014;260:848-56.

35. Nguyen AH, Toste PA, Farrell JJ, Clerkin BM, Williams J, Muthusamy VR, et al. Current recommendations for surveillance and surgery of intraductal papillary mucinous neoplasms may overlook some patients with cancer. J Gastrointest Surg. 2015;19:848-56

36. Shimizu Y, Kanemitsu Y, Sano T, Senda Y, Mizuno N, Yamao K, et al. A nomogram for predicting the probability of carcinoma in patients with intraductal papillary mucinous neoplasm. World $\mathrm{J}$ Surg. 2010;34:2932-8.

37. Ohtsuka T, Matsunaga T, Kimura H, Watanabe Y, Tamura K, Ideno N, et al. Role of pancreatic juice cytology in the preoperative management of intraductal papillary mucinous neoplasm of the pancreas in the era of international consensus guidelines. World J Surg. 2014;38:2994-3001

38. Hirono S, Tani M, Kawai M, Okada K, Miyazawa M, Shimizu A, et al. The carcinoembryonic antigen level in pancreatic juice and mural nodule size are predictors of malignancy for branch duct type intraductal papillary mucinous neoplasms of the pancreas. Ann Surg. 2012;255:517-22.

39. Hayakawa H, Fukasawa M, Sato T, Takano S, Kadokura M, Shindo H, et al. Carcinoembryonic antigen level in the pancreatic juice is effective in malignancy diagnosis and prediction of future malignant transformation of intraductal papillary mucinous neoplasm of the pancreas. J Gastroenterol. 2019;54:1029-37.

40. Kawai M, Uchiyama K, Tani M, Onishi H, Kinoshita H, Ueno $\mathrm{M}$, et al. Clinicopathological features of malignant intraductal papillary mucinous tumors of the pancreas. Arch Surg. 2004;139:188-92.

41. Maire F, Voitot H, Aubert A, Palazzo L, O'Toole D, Couvelard A, et al. Intraductal papillary mucinous neoplasms of the pancreas: performance of pancreatic fluid analysis for positive diagnosis and the prediction of malignancy. Am J Gasetroenterol. 2008; 103:2871-7.

42. Hirooka Y, Goto H, Itoh A, Hashimoto S, Niwa K, Ishikawa H, et al. Case of intraductal papillary mucinous tumor in which endosonography-guided fine-needle aspiration biopsy caused dissemination. J Gastroenterol Hepatol. 2003;18:1323-7.

43. Ahmed K, Sussman JJ, Wang J, Schmulewitz N. A case of EUSguided FNA-related pancreatic cancer metastasis to the stomach. Gastrointest Endosc. 2011;74:231-3.

44. Shimizu Y, Hijioka S, Hirono S, Kin T, Ohtsuka T, Kanno A, et al. New model for predicting malignancy in patients with intraductal papillary mucinous neoplasm. Ann Surg. 2018. https://doi. org/10.1097/SLA.0000000000003108.

45. Jang JY, Park T, Lee S, Kim Y, Lee SY, Kim SW, et al. Proposed nomogram predicting the individual risk of malignancy in the patients with branch duct type intraductal papillary mucinous neoplasms of the pancreas. Ann Surg. 2017;266:1062-8.

46. Attiyeh Marc A, Fernández-del Castillo C, Efishat MA, Eaton AA, Gönen M, Batts R, et al. Development and validation of a multi-institutional preoperative nomogram for predicting grade of dysplasia in intraductal papillary mucinous neoplasms (IPMNs) of the pancreas. A report from the Pancreatic Surgery Consortium. Ann Surg. 2018;267:157-63.

47. Shimizu Y, Yamaue H, Maguchi H, Yamao K, Hirono S, Osanai $\mathrm{M}$, et al. Predictors of malignancy in intraductal papillary mucinous neoplasm of the pancreas: analysis of 310 pancreatic resection patients at multiple high-volume centers. Pancreas. 2013;42:883-8.

48. Hirono S, Kawai M, Okada KI, Miyazawa M, Shimizu A, Kitahata $\mathrm{Y}$, et al. Factors associated with invasive intraductal papillary mucinous carcinoma of the pancreas. JAMA Surg. 2017. https:// doi.org/10.1001/jamasurg.2016.5054.

49. Turrini O, Waters JA, Schnelldorfer T, Lillemoe KD, Yiannoutsos $\mathrm{CT}$, Farnell MB, et al. Invasive intraductal papillary mucinous neoplasm: predictors of survival and role of adjuvant therapy. HPB. 2010;12:447-55.

50. McMillan MT, Lewis RS, Drebin JA, Teitelbaum UR, Lee MK, Roses RE, et al. The efficacy of adjuvant therapy for pancreatic invasive intraductal papillary mucinous neoplasm (IPMN). Cancer. 2016;122:521-33.

51. Duconseil P, Périnal J, Autret A, Adham M, Sauvanet A, Chiche $\mathrm{L}$, et al. Resectable invasive IPMN versus sporadic pancreatic adenocarcinoma of the head of the pancreas: should these two different diseases receive the same treatment? A matched comparison study of the French Surgical Association (AFC). EJSO. 2017;43:1704-10.

52. Kaiser J, Fritz S, Kkauss M, Bergmann F, Hinz U, Strobel O, et al. Enucleation: a treatment alternative for branch duct intraductal papillary mucinous neoplasms. Surgery. 2017;161:602-10.

53. Sauvanet A, Gaujoux S, Blanc B, Couvelard A, Dokmak S, Vullierme MP, et al. Parenchyma-sparing pancreatectomy for presumed 
noninvasive intraductal papillary mucinous neoplasms of the pancreas. Ann Surg. 2014;260:364-71.

54. Faitot F, Gaujoux S, Barbier L, Novaes M, Dokmak S, Aussilhou B, et al. Reappraisal of pancreatic enucleations: a single-center experience of 126 procedures. Surgery. 2015;158:201-10.

55. Hirono S, Tani M, Kawai M, Ina S, Nishioka R, Miyazawa M, et al. A central pancreatectomy for benign or low-grade malignant neoplasms. J Gastrointest Surg. 2009;13:1659-65.

56. Sauvanet A, Gaujoux S, Blanc B, Couvelard A, Dokmak S, Vullierme MP, et al. Parenchyma-sparing pancreatectomy for presumed noninvasive intraductal papillary mucinous neoplasms of the pancreas. Ann Surg. 2014;260:364-71.

57. Marangos IP, Buanes T, Røsok BI, Kazaryan AM, Rosseland AR, Grzyb K, et al. Laparoscopic resection of exocrine carcinoma in central and distal pancreas results in a high rate of radical resections and long postoperative survival. Surgery. 2012;151:517-23.

58. Nakamura $M$, Nakashima $H$. Laparoscopic distal pancreatectomy and panreatoduodenectomy: is it worthwhile? A meta-analysis of laparoscopic pancreatectomy. J Hepatobiliary Pancreat Sci. 2013;20:421-8.

59. Konstantinidis IT, Jutric Z, Eng OS, Warner SG, Melstrom LG, Fong Y, et al. Robotic total pancreatectomy with splenectomy: technique and outcomes. Surg Endosc. 2018;32:3691-6.

60. Hong D, Liu Y, Peng S, Sun X, Wang Z, Cheng J, et al. Binding pancreaticogastrostomy in laparoscopic central pancreatectomy: a novel technique in laparoscopic pancreatic surgery. Surg Endosc. 2016;30:715-20.

Publisher's Note Springer Nature remains neutral with regard to jurisdictional claims in published maps and institutional affiliations. 